\title{
PERFIL NUTRICIONAL DE CRIANÇAS INTERNADAS EM UM HOSPITAL PÚBLICO DE CAMPINA GRANDE - PB
}

\author{
NUTRITIONAL PROFILE OF CHILDREN HOSPITALIZED IN A PUBLIC \\ HOSPITAL IN CAMPINA GRANDE - PB
}

\author{
Davyson Barbosa Duarte ${ }^{1}$ \\ Iris Damião Macena ${ }^{2}$ \\ Mayana Kelly Tavares de Souza ${ }^{3}$ \\ Wanessa de Oliveira Mendonça ${ }^{4}$ \\ Lidiane da Conceição Nóbrega de Araújo ${ }^{5}$
}

\begin{abstract}
RESUMO: OBJETIVO: Este trabalho objetivou avaliar o estado e risco nutricional de crianças internadas em um hospital público de Campina Grande - PB. MÉTODO: Realizou- se uma pesquisa descritiva, qualitativa e quantitativa, com uma amostra de 30 crianças, com faixa etária de 2 a 10 anos, admitidas em até 48 horas na enfermaria pediátrica do Hospital de Emergência e Trauma Dom Luiz Gonzaga Fernandes. Foi realizada avaliação antropométrica para classificação do estado nutricional segundo os padrões da Organização Mundial da Saúde (OMS) e procedeu-se com aplicação da ferramenta de triagem nutricional STRONGkids. Para todos os parâmetros antropométricos abordados, a maioria da amostra apresentouse eutrófica. Entretanto, o STRONGkids classificou $70 \%(n=21)$ das crianças com risco nutricional moderado. Ademais, o diagnóstico médico mais frequente foi fratura óssea $33,33 \%(n=10)$ e apendicectomia $26,7 \%(n=8)$, podendo considerar que esse quadro e demais situações relacionadas estão associadas ao risco nutricional moderado.RESULTADOS: Portanto, o fato do estado nutricional não se encontrar afetado na admissão hospitalar não descarta a possibilidade do desenvolvimento da desnutrição durante a internação. CONCLUSÃO: Nesse contexto, sugere-se o acompanhamento desse estado, bem como a associação de métodos objetivos e subjetivos para uma abordagem nutricional completa.
\end{abstract}

Palavras chave: Antropometria. Avaliação nutricional. Hospitais. Pediatria.

\footnotetext{
${ }^{1}$ Pós-graduando em Nutrição Clínica Funcional e Esportiva com Ênfase em Nutrigenômica pelo Centro Universitário UNIFACISA.

${ }^{2}$ Pós-graduada em Nutrição Ortomolecular pela Faculdade Instituto Brasil de Ensino - IBRA.

${ }^{3}$ Nutricionista do Hospital de Emergência e Trauma Dom Luiz Gonzaga Fernandes, Esp. em Nutrição Clínica e Professora do curso de Bacharelado em Nutrição do Centro Universitário Maurício de Nassau - UNINASSAU.

${ }^{4}$ Graduada em Nutrição pelo Centro Universitário Maurício de Nassau - UNINASSAU.

${ }^{5}$ Pós-graduanda em Nutrição Esportiva pelo Centro Universitário de João Pessoa - UNIPÊ.
} 
ABSTRACT: OBJECTIVE: This study aimed to assess the nutritional status and risk of children admitted to a public hospital in Campina Grande - PB. METHOD: A descriptive, qualitative and quantitative research was carried out with a sample of 30 children, aged 2 to 10 years, admitted within 48 hours to the pediatric ward of the Hospital de Emergência e Trauma Dom Luiz Gonzaga Fernandes. An anthropometric assessment was carried out to classify nutritional status according to the standards of the World Health Organization (WHO) and proceeded with the application of the nutritional screening tool STRONGkids. For all the anthropometric parameters addressed, most of the sample was eutrophic. However, STRONGkids classified $70 \%(n=21)$ of children with moderate nutritional risk. In addition, the most frequent medical diagnosis was bone fracture $33,33 \%(n=10)$ and appendectomy $26,7 \%(n=8)$, which may consider that this condition and other related situations are associated with moderate nutritional risk. RESULTS: Therefore, the fact that the nutritional status is not affected on hospital admission does not rule out the possibility of the development of malnutrition during hospitalization. CONCLUSION: In this context, the monitoring of this state is suggested, as well as the association of objective and subjective methods for a complete nutritional approach.

Keywords: Anthropometry. Hospitals. Nutritional assessment. Pediatrics. 


\section{INTRODUÇÃO}

A infância trata-se de um estágio de vida importante para o desenvolvimento do ser humano, no que se refere aos aspectos biológicos, psicológicos, sociais e intelectuais. Assim, o surgimento de doenças nesse período da vida pode implicar em danos como trauma, atraso ou interrupção de desenvolvimento e crescimento infantil. Desse modo, uma alimentação adequada qualitativamente e quantitativamente é indispensável para suprir a necessidade de energia e nutrientes que o organismo necessita para desempenhar satisfatoriamente suas funções, bem como implicar na manutenção da saúde (BORTOLOTE; BRÊNTAS, 2008; BOEIRA et al., 2012; FREIBERG; FERREIRA, 2008).

$O$ atendimento inapropriado das necessidades energéticas do indivíduo pode resultar em distúrbios alimentares, sendo a desnutrição e a obesidade aqueles que mais acometem o público infantil, podendo comprometer gravemente a saúde dessa população. A desnutrição infantil é um dos problemas de saúde pública de maior relevância, devido às consequências no crescimento e desenvolvimento, além da maior vulnerabilidade a doenças infecciosas. Compreende-se que esse quadro, bem como o de obesidade, associam-se de forma direta a inúmeras complicações que refletem no aumento da morbidade e tempo de internação (TALMA; MIRANDA, 2013; JOOSTEN et al., 2010; PAWELLEK; DOKOUPIL; KOLETZKO, 2008; SIMÕES et al., 2010).

No que se refere ao público infantil, os mais suscetíveis à hospitalização estão as com idade inferior a seis meses, baixo peso ao nascer e prematuridade, condições essas relacionadas à desnutrição. Além disso, inúmeros fatores estão associados a esse desequilíbrio alimentar, como o baixo nível socioeconômico, perda de apetite, absorção inadequada, presença de patologias prévias e aumento das necessidades energéticas. Dessa maneira, as alterações nutricionais ocasionadas possuem grande importância para o paciente, especialmente em crianças, no qual as necessidades nutricionais são distintas das do adulto. 
(CORREIA; CAMPOS, 2003; FALBO; BEZERRA, 2002; WAITZBERG; CAIAFFA; CORREIA, 2001; WAITZBERG; CORREIA, 2000).

No Brasil, a incidência de mortes por desnutrição infantil hospitalar grave corresponde a aproximadamente 20\%. Geralmente, observa-se que esses óbitos ocorrem nas primeiras 48 horas de internação, demonstrando possíveis falhas do atendimento hospitalar na admissão dessas crianças. Essa patologia afeta negativamente a evolução clínica de crianças hospitalizadas acarretando em aumento ou risco de doenças infecciosas, retardo em processos de cicatrização, edemas gerados por deficiência proteica, redução do trânsito intestinal, propensão ao choque e diminuição da barreira imunológica. Além disso, estudos apontam que o tratamento de pacientes desnutridos custa quatro vezes mais, quando comparado ao de um paciente com estado nutricional adequado. Nesse âmbito, ressalta-se a importância no reconhecimento precoce de crianças em risco nutricional elevado, permitindo intervir com tratamento dietoterápico individualizado e eficaz no impedimento do agravo do estado nutricional ou na recuperação do mesmo (BRASIL, 2005; DUCHINI et al., 2010; KARATEKE et al., 2013; HULST et al., 2010; MEHTA et al., 2013; MOEENI; WALLS; DAY, 2012).

A avaliação do estado nutricional de indivíduos, em especial de crianças pode ser considerada um ótimo indicador para investigar a conformidade do seu estado de saúde, verificando se o mesmo está sendo prejudicado por quadro de doença e/ou condição socioeconômico desfavorável. O método mais comumente utilizado para análise, classificação e acompanhamento do estado nutricional é a avaliação antropométrica, ressalta-se que esta pode ser associada à aplicação de ferramentas de triagem, dentre as quais destaca-se o "STRONGkids", que trata-se de um instrumento que aponta o risco de desconformidades nutricionais (CARVALHO et al., 2013; HEYWARD; STOLARCZYK, 2000; MELLO, 2002; SIGULEM; DEVINCENZI; LESSA, 2000).Deste modo, este trabalho teve como objetivo avaliar o estado e risco nutricional de crianças internadas em um hospital público de Campina Grande - PB. 


\section{MATERIAL E MÉTODOS}

Esse estudo trata-se de uma pesquisa descritiva de caráter qualitativo e quantitativo, realizada na ala pediátrica do Hospital de Emergência e Trauma Dom Luiz Gonzaga Fernandes, localizado no município de Campina Grande - PB.

A coleta de dados ocorreu no mês de novembro de 2018, na qual foi possível obter uma amostra, não probabilística e aleatória, de 30 crianças. Foram incluídas aquelas com idade de dois a dez anos, admitidas na enfermaria pediátrica da referida instituição, que não tivessem mais de 48 horas de internação hospitalar e com capacidade de deambular.

Para realização das medidas antropométricas utilizou-se balança digital GTech, BALGL10, em vidro temperado, transparente, com capacidade máxima de 150 $\mathrm{kg}$, para aferição do peso; e medidor de estatura estadiômetro portátil, sendo estas aferidas de acordo as técnicas de Lohman et al. (1988). Os dados obtidos foram analisados segundo os parâmetros indicados pelo Ministério da Saúde avaliando-se os índices Peso/ldade e Estatura/ldade, de acordo com o sexo, por meio das curvas de crescimento (nas quais constam percentis e escores $z$ ) estabelecidas pela Organização Mundial da Saúde (2006), para crianças com faixa etária entre zero a cinco anos, bem como pelo padrão OMS (2007), para crianças com idade superior a cinco anos.

O nível de risco nutricional das crianças inclusas na pesquisa foi verificado através de uma adaptação do questionário STRONGkids, de Talma e Miranda (2013), o qual identifica situações de risco por meio do colhimento de informações como presença de doença de alto risco ou previsão de cirurgia de grande porte, redução da ingestão alimentar, ocorrência de diarreia e vômito, e perda ou dificuldade de ganho de peso, sendo o resultado final obtido por meio de um sistema de somatório, onde atribui-se pontos a respostas positivas, e faz-se a seguinte classificação: Alto risco: 4-5 pontos; Médio risco: 1-3 pontos; Baixo risco: 0 pontos. Em caráter complementar, foram colhidos dados dos prontuários dos pacientes, a fim de obter informações como idade e diagnóstico médico. 
Por fim, realizou-se a análise descritiva dos dados, determinando-se a frequência relativa percentual de cada variável estudada. Posteriormente, os dados obtidos foram tabulados no programa Excel, do Microsoft Office 2010, sendo os resultados finais apresentados em tabelas e gráficos para melhor entendimento.

O projeto foi aprovado pelo Comitê de Ética e Pesquisa do Hospital Universitário Alcides Carneiro conforme resolução 466/12 do Conselho Nacional de Saúde, sob número de parecer: CAE 95388618.4.0000.5182.

\section{RESULTADOS E DISCUSSÃO}

Para o presente estudo, participaram 30 crianças hospitalizadas, as quais apresentaram idade entre 2 e 10 anos, dentre elas, 53,33\% ( $n=16)$ eram do sexo masculino e 46,67\% ( $n=14)$ do sexo feminino (Tabela 1). Dados semelhantes foram descritos no estudo de Pedersoli et al. (2017), com pacientes internados em um hospital infantil, o qual constituiu-se de 21 meninos e 18 meninas, correspondendo a $54 \%$ e $46 \%$, respectivamente. Uma das justificativas possíveis para o predomínio do gênero masculino nas amostras seria a maior concessão de liberdade desses para ações, bem como brincadeiras, em razão aos aspectos socioculturais relacionados as diferenças de gênero, resultando em uma maior vulnerabilidade a infecções e traumas (SIMÕES et al., 2010).

Tabela 1: Distribuição da amostra de crianças quanto ao gênero.

\begin{tabular}{l|c|c}
\hline Sexo & $\mathbf{N}$ & $\%$ \\
\hline Masculino & 16 & 53,33 \\
Feminino & 14 & 46,67 \\
\hline TOTAL & 30 & 100 \\
\hline
\end{tabular}

Fonte: Dados da pesquisa (2018).

A avaliação nutricional é um ótimo instrumento para a determinação do estado nutricional, em especial do público infantil, visto que distúrbios nutricionais nessa fase podem acarretar em graves complicações no desenvolvimento desse 
grupo (MELLO, 2002). Entretanto, a sua identificação muitas vezes não é mencionada nos prontuários desses pacientes, o que constitui uma negligência. Este fato foi visto durante a coleta de dados dos participantes desta pesquisa. Sendo também evidenciado por Huber e Vinholes (2015), que na realização do seu estudo, com crianças de até 12 anos incompletos, internadas na enfermaria pediátrica do Hospital Nossa Senhora da Conceição, do município de Tubarão - SC, constataram que $92,8 \%$ dos diagnósticos emitidos não possuíam essa informação, desse modo, apenas $7,2 \%$ dos pacientes possuíam o estado nutricional declarado em seus prontuários.

Os parâmetros antropométricos (E/I e P/I), dispostos na Tabela 2, demonstraram uma maior proporção de crianças com estatura adequada e eutróficas para idade, respectivamente 90\% $(n=27)$ e 96,67\% (n=29). Valores similares são vistos na literatura através de Santos et al. (2014), que constatou índices de estatura para idade prevalentemente adequados $(89,19 \% ; n=33)$ e baixa estatura $(10,81 \% ; n=4)$; como também verificou peso para idade, classificando $91,89 \%(n=34)$ como eutrofia e $8,11 \%(n=3)$ baixo peso, em uma amostra de 37 crianças internadas no Hospital Regional Justino Luz, no município de Picos - PI.

Tabela 2: Estado nutricional segundo os índices peso/idade e estatura/idade.

\begin{tabular}{l|c|c}
\hline \multicolumn{3}{|c}{ Peso/Idade } \\
\hline Estado nutricional & $\mathbf{N}$ & $\%$ \\
\hline Magreza III & 0 & 0 \\
Magreza II & 0 & 0 \\
Magreza I & 0 & 0 \\
Eutrofia & 29 & 96,67 \\
Sobrepeso & 1 & 3,33 \\
Obesidade & 0 & 0 \\
\hline
\end{tabular}

Estatura/ldade

\begin{tabular}{l|l|ll}
\hline Estado nutricional & \multicolumn{1}{|c|}{$\mathbf{N}$} & \multicolumn{1}{c}{$\%$} \\
\hline Baixa estatura & 3 & 10 & \\
Estatura adequada & 27 & 90 &
\end{tabular}

Fonte: Dados da pesquisa (2018).

Outros autores evidenciam resultados aproximados aos da presente pesquisa, tais como Pedersoli et al. (2017), que observou prevalência de adequação 
para $E / I$ em $92,30 \%(n=36)$ e baixa estatura em 7,7\% $(n=3)$ e para $P / I$, eutrofia em $82 \%(n=34)$ da amostra. Ademais, Huber e Vinholes (2015), Lima e Tiengo (2012) e Magalhães et al. (2013) apresentam também em seus estudos com crianças hospitalizadas, maior incidência do estado de eutrofia para esse último índice, com valores de 64,40\% ( $n=116), 63 \%(n=53)$ e 58,7\% $(n=81)$, respectivamente. Dessa forma, observou-se que independentemente do número da amostra, o estado nutricional mais recorrente foi o mesmo.

Os índices de P/l e E/l utilizados para a classificação do estado nutricional, são amplamente usados para avaliar o crescimento linear e expressar a situação global da qualidade de vida da criança (HUBER; VINHOLES, 2015). Dessa maneira, a partir dos dados e interpretações obtidos desses parâmetros, verificou-se que a amostra se apresenta, majoritariamente, em eutrofia e com estatura adequada, corroborando com os encontrados na literatura. Assim, este fato pode ser justificado, principalmente pelo declínio de $73,4 \%$ da desnutrição em crianças menores de 5 anos, entre o período de 1996-2006, caracterizando ao decorrer das décadas o antagonismo da redução desse distúrbio com 0 aumento das medidas antropométricas, denominado de transição nutricional (LIMA et al., 2010).

Corroborando como os resultados obtidos nessa pesquisa, Campos et al. (2015), Mahdavi, Safaiyan e Ostadrahimi (2009) e Spagnuolo et al. (2013) em seus estudos com crianças hospitalizadas, com faixas etárias de 4 a 8,9 anos, 2 a 12 anos e 1 a 18 anos, respectivamente, demonstraram predominância de estado nutricional satisfatório, na admissão hospitalar, quando avaliados por medidas antropométricas. Entretanto, esse método de avaliação, considerado objetivo, detecta apenas indivíduos que já são acometidos por desnutrição, assim não avaliando os que são submetidos ao risco de instalação desse distúrbio nutricional.

Em contrapartida, vale ressaltar a importância na detecção prévia de crianças com risco nutricional, visto que a perda de peso é frequente durante o período de hospitalização, por possíveis fatores como anorexia, dificuldade de deglutição, náuseas, medicamentos prescritos, não aceitação da dieta, necessidade energética aumentada e digestão e absorção de nutrientes prejudicadas. Fatores estes relacionados a doença de base ou até mesmo ao âmbito hospitalar, que se mostra desfavorável a recuperação de alguns pacientes devido influência psicológica. Outro 
fator que pode estar associado a esse quadro é o jejum a que os pacientes são submetidos em pré-operatórios, pois o mesmo é estendido com frequência, na maioria das vezes, a mais de 8 horas, podendo permanecer em média 16 horas ou mais até o momento da anestesia, devido ao atraso ou adiamento das cirurgias (FLORES; KIK, 2013; LEANDRO et al., 2004; SERMET-GAUDELUS et al., 2000).

Dessa forma, segundo Silva e Tiengo (2014), é indicada para avaliar o estado e risco nutricional de crianças, a associação entre métodos objetivos e subjetivos,para uma interpretação mais fidedigna. Nesse contexto, em âmbito hospitalar, destaca-se a ferramenta de triagem STRONGkids, sendo considerada de fácil e rápida aplicação. A partir de sua aplicação, constatou-se na presente pesquisa que $70 \%(n=21)$ das crianças encontravam-se com moderado risco nutricional,30\% $(n=9)$ com baixo risco e nenhuma apresentou risco elevado (Figura 1).

Figura 1: Número (n) e percentual (\%) de risco nutricional segundo o STRONGkids.

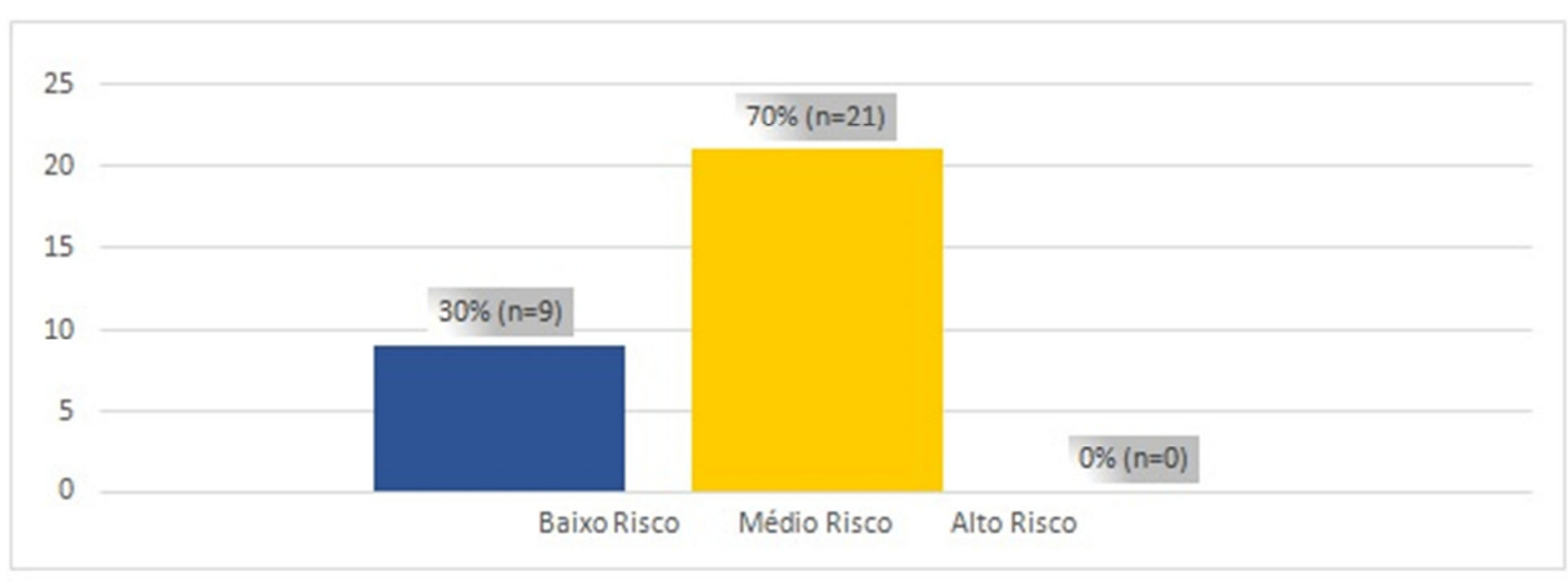

Em um estudo similar, Campos et al. (2015) obteve resultados semelhantes que ratificam os encontrados nessa pesquisa, no qual avaliou 317 crianças com 4 a 8,9 anos, em um hospital pediátrico de Porto Alegre - RS, identificando 67,5\% ( $n=214)$ com médio risco e $24,6 \%(n=78)$ baixo risco. Além deste, a nível mundial pode-se observar valores aproximados com os mencionados, através dos autores Costa et al. (2017) por meio de uma amostra de 63 crianças internadas em um hospital português, no qual $58,7 \%(n=37)$ enquadraram-se em grupo de risco 
moderado, $38,1 \%(n=24)$ e $3,2 \%(n=2)$ respectivamente, baixo e elevado risco nutricional. Dados semelhantes foram encontrados por Spagnuolo et al. (2013), que detectou em 144 crianças italianas hospitalizadas, 53\% $(n=76)$ com médio risco, $32 \%(n=46)$ baixo risco e $15 \%(n=22)$ alto risco, sendo que ambos incluem participantes com faixa etária entre 1 a 18 anos.

A literatura evidencia que ao comparar os dados obtidos por meio desse instrumento com os índices antropométricos, verifica-se que não há uma relação significativa entre esses parâmetros, fato visto também no presente estudo, onde segundo a antropometria $90 \%$ e $96,67 \%$ das amostras encontram-se, respectivamente, com estatura e peso para idade adequados (Tabela 2). Em contrapartida, de acordo com o STRONGkids $70 \%$ das crianças apresentaram risco nutricional moderado (Figura 1). Logo, essa premissa pode ser justificada pela sensibilidade elevada desse último instrumento em identificar risco nutricional, uma vez que a existência de uma única pontuação já aponta possibilidades de alterações no estado nutricional, sendo considerada como médio risco, conferindo assim, tal característica (CAMPOS et al., 2015; WISKIN et al., 2012).

Quanto à causa de internação das crianças, o diagnóstico médico mais frequente foi fratura óssea $(33,33 \% ; n=10)$, seguido de apendicectomia $(26,7 \%$; $\mathrm{n}=8$ ), como demonstrado na Tabela 3. No entanto, esses resultados divergem dos encontrados em estudos de Rodrigues et al. (2016) e Magalhães et al. (2013), realizados com cerca de 200 pacientes pediátricos de hospitais universitários de Minas Gerais e do Rio de Janeiro, respectivamente, nos quais as doenças respiratórias foram a causa mais prevalente de hospitalização, correspondendo, nessa mesma ordem a 33,2\% $(n=62)$ e $29,5 \%(n=59)$. Huber e Vinholes (2015), também encontraram que $35,63 \%(n=40)$ apresentaram diagnóstico de pneumonia e $11,00 \% \quad(n=12)$ de bronquiolite, totalizando maior prevalência de doenças respiratórias $(46,63 \%)$. Vale ressaltar que, no presente estudo a maior prevalência do diagnóstico foi de fraturas ósseas, devido a principal especialidade do hospital ser trauma. 
Tabela 3: Diagnóstico médico das crianças estudadas.

\begin{tabular}{l|c|c}
\hline Diagnóstico & $\mathbf{N}$ & $\%$ \\
\hline Abdome agudo & 3 & 10 \\
Afogamento & 1 & 3,33 \\
Apendicectomia & 8 & 26,7 \\
Celulite facial & 1 & 3,33 \\
Fratura óssea & 10 & 33,33 \\
Infeç̧ão do Trato Urinário & 1 & 3,33 \\
Insuficiência respiratória & 1 & 3,33 \\
Lítiase renal & 1 & 3,33 \\
Obstrução Intestinal & 1 & 3,33 \\
Pneumonia & 2 & 6,66 \\
Traumatismo Craniano Encefálico & 1 & 3,33 \\
\hline
\end{tabular}

Fonte: Dados da pesquisa (2018).

Em um estudo realizado por Ribeiro, Alves e Fatal (2018) com crianças de 0 a 9 anos e 11 meses, hospitalizadas em uma enfermaria pediátrica de um hospital de grande porte em Salvador - BA, embora tenha detectado valores que corroboram com a literatura de maior incidência de doenças respiratórias, como broncopneumonia, pneumonia e outras afecções do sistema respiratório $(35,4 \%)$, também observaram que a segunda maior causa de internações eram de apendicectomia, correspondendo a $20,8 \%$ dos motivos de internações. Fato este, semelhante ao encontrado na presente pesquisa. Simões et al. (2010), também encontrou na sua pesquisa que entre 749 crianças e adolescentes da enfermaria de cirurgia do Hospital de São Paulo, 131 dos mesmos foram submetidos à cirurgia geral, no qual a mesma observou que a cirurgia pediátrica geral apresentava grandes números de hospitalizações para tratamento de apendicite aguda.

Observou-se durante o período de coleta de dados que as fraturas ósseas podem influenciar negativamente no estado nutricional, uma vez que o atraso para realização desses procedimentos acaba aumentando os dias de internação hospitalar e, consequentemente, expondo os pacientes a outros riscos hospitalares, bem como a baixa ingestão da dieta ofertada, comum nesse ambiente. Além disso, uma vez que a instituição trata-se de um hospital de emergência e trauma, muitas vezes o paciente é submetido ao jejum pré-operatório desnecessário, pois 
admissões emergenciais são prioritárias, e se ocorrerem durante esse período de espera, fazem com que a cirurgia deste seja suspensa.

Dessa maneira, a resposta metabólica desencadeada pelo trauma sofrido acaba sendo potencializada com esses longos períodos de jejum antes da realização da cirurgia, em virtude do aumento da atividade dos hormônios contrarreguladores (insulina e glucagon), bem como da produção elevada de mediadores inflamatórios, resultando em vários efeitos, tais como aumento da resistência à insulina, lipólise, proteólise muscular e até mesmo podendo levar a inflamação sistêmica, dependendo do porte da operação (FLORES; KIK, 2013; NYGREN, 2006). Visto que, o estresse cirúrgico proporcionado nas intervenções de grande porte intensifica a degradação de macromoléculas (catabolismo), para liberação de energia que satisfaça a resposta ao processo. Assim, esses fatores podem afetar o estado nutricional dos pacientes, que em especial ao do público infantil, proporcionam maior déficit nutricional e aumento no tempo de hospitalização (BOTTONI, 2002).

Dessa forma, foram criadas várias diretrizes baseadas em estudos, que demonstraram que é possível a abreviação do jejum mesmo em cirurgias de grande porte, assim destaca-se o projeto ACERTO (Aceleração da Recuperação Total Pósoperatória), que preconiza a diminuição do tempo de jejum, para sólidos de 6-8h e de $2 \mathrm{~h}$ para líquidos contendo maltodextrina, ambos antes da anestesia para procedimentos eletivos. Embora o jejum depois da meia noite seja uma prática difícil de ser alterada, estudos apontam a crescente aderência dessas novas recomendações pelos cirurgiões e anestesistas. (DE-AGUILAR-NASCIMENTO et al., 2017; GIULIANE; MCARTHUR, GREENWOOD, 2015; NYGREN et al., 2012).

Em relação aos pacientes submetidos à apendicectomia, foi observado que a evolução dietoterápica lenta, no que diz respeito a consistência e conteúdo energético da dieta durante o pós-operatório, exigida por tratar-se de um procedimento que envolve o trato gastrointestinal, também pode vir a prejudicar o diagnóstico nutricional. Dessa maneira, pode-se considerar que essa e as demais situações mencionadas estão associadas ao risco nutricional moderado. 


\section{CONCLUSÃO}

Diante dos resultados apresentados, observou-se uma maior prevalência de eutrofia através da antropometria, porém, este fato não descartou a constatação de risco nutricional moderado através do STRONGkids, o que indica que apesar do estado nutricional não encontrar-se afetado no momento da admissão, isso não exclui a possibilidade do desenvolvimento de desnutrição durante o período de hospitalização. Nesse contexto, sugere- se o acompanhamento desse estado durante a internação, bem como, a associação de métodos objetivos, como os índices antropométricos e subjetivos, como o STRONGkids para uma abordagem nutricional completa.

Ressalta-se, ainda, que a realização precoce da avaliação do estado nutricional e a identificação de riscos, alerta para a adequação do tratamento dietoterápico individualizado caso necessário, prevenindo agravos e propiciando um melhor prognóstico para o paciente. Dessa maneira, enfatiza-se a importância da determinação do estado nutricional das crianças, o qual muitas vezes encontra-se negligenciado no prontuário das mesmas.

\section{REFERÊNCIAS BIBLIOGRÁFICAS}

BOEIRA, G.; MANFIO, F.; SILVA, R. M.; IOP, S.; PEDROTTI, T. Perfil nutricional de crianças hospitalizadas. UNIFRA Evento/Sepse, 2012 [Internet]. Acesso em: 02 de Abril de 2018. Disponível em: http://www.unifra.br/eventos/sepe2012/Trabalhos/6643.pdf.

BORTOLETE, G. S.; BRÊTAS, J. R. S. O ambiente estimulador ao desenvolvimento da criança hospitalizada. Revista da Escola de Enfermagem da USP, São Paulo, v. 42, n. 3, p. 422-429, 2008.

BOTTONI, A. Cirurgia e trauma. In: Cuppari, L. Nutrição clínica no adulto. Barueri: Manole, p. 319-342, 2002. BRASIL, M. S. Manual de Atendimento da Criança com Desnutrição Grave em Nível Hospitalar. Série A. Normas e Manuais Técnicos. Secretaria de Atenção à Saúde. Coordenação Geral da Política de Alimentação e Nutrição - Brasília, 2005.

CAMPOS, L. S. K.; NEUMANN, L. D.; RABITO, E. I.; MELLO, E. D.; VALLANDRO, J. P. Avaliação do risco nutricional em crianças hospitalizadas: uma comparação da avaliação subjetiva global pediátrica e triagem nutricional STRONGkids com os indicadores 
antropométricos. Scientia Medica, v. 25, n. 3, p. 1-8, 2015.

CARVALHO, F. C.; LOPES, C. R.; VILELA, L. C.; VIEIRA, M. A.; RINALDI, A. E. M.; CRISPIM, C. A. Tradução e adaptação cultural da ferramenta Strongkids para triagem do risco de desnutrição em crianças hospitalizadas. Revista Paulista de Pediatria, [S.I.], v. 31, n. 2, p. 159-165, 2013.

CORREIA, M. I. T. D.; CAMPOS, A. C. L. Prevalence of Hospital Malnutrition in Latin America: The Multicenter ELAN Study. Revista de Nutrição, Belo Horizonte, v. 19, n. 10, p. 823- 825, 2003.

COSTA, C.; MATOS, C.; CÂNDIDO, C.; GASPAR, E. Avaliação do risco nutricional e caracterização do estado nutricional de crianças internadas. Acta Portuguesa de Nutrição, [S.I], v. 10, p. 18-22, 2017.

DE-AGUILAR-NASCIMENTO, J. E.; SALOMÃO, A. B.; WAITZBERG, D. L.; DOCKNASCIMENTO, D. B.; CORREIA, M. I. T. D.; CAMPOS, A. C. L.; CORSI, P. R.; PORTARI FILHO, P. E.; CAPOROSSI, C. Diretriz ACERTO de intervenções nutricionais no perioperatório em cirurgia geral eletiva. Revista do Colégio Brasileiro de Cirurgiões, [S.I.], v. 44, n. 6, p. 633-648, 2017.

DUCHINI, L.; JORDÃO, A. A.; BRITO, T.; DIEZ-GARCIA, R. W. Avaliação e monitoramento do estado nutricional de pacientes hospitalizados: uma proposta apoiada na opinião da comunidade científica. Revista de Nutrição, Campinas, v. 23, n. 4, p. 513-522, 2010.

FALBO, A. R.; BEZERRA A. J. G. Desnutrição grave: alguns aspectos clínicos e epidemiológicos de crianças hospitalizadas no Instituto materno infantil de Pernambuco (IMIP), Brasil. Cadernos de Saúde Pública, Rio de Janeiro, v. 18, n. 1, p. 1473-1477, 2002.

FLORES, P. F.; KIK, R. M. Jejum pré-operatório em pacientes hospitalizados. Revista Ciência \& Saúde, Porto Alegre, v. 6, n. 3, p. 214-221, 2013.

FREIBERG, C. K.; FERREIRA, R. T. Alimentação do pré-escolar. In: GALISA, M. S.; ESPERANÇA, L. M. B.; SÁ, N. G. Nutrição: conceitos e aplicações. São Paulo: M. Books, p. 141-147, 2008.

GIULIANI, S.; MCARTHUR, A.; GREENWOOD, J. Preoperative fasting among burns patients in an acute care setting: a best practice implementation project. JBI Database of Systematic Reviews \& Implementation Reports, [S.I.], v. 13, n. 11, p. 235-253, 2015.

HEYWARD, V. H.; STOLARCZYK, L. M. Avaliação da composição corporal aplicada. Rio de Janeiro: Manole, p. 243, 2000.

HUBER, E.; VINHOLES, D. B. Estado nutricional de crianças internadas na pediatria de um hospital terciário. Arquivos de Ciências da Saúde, [S.I.]. v. 22, n. 3, p. 91-95, 2015.

HULST, J. M.; ZWART, H.; HOP, W. C.; JOOSTEN, K. F. Dutch national survey to test the STRONGkids nutritional risk screening tool in hospitalized children. Clinical Nutrition, [S.I.], v. 29, p. 106-111, 2010.

JOOSTEN, K. F.; ZWART, H.; HOP, W. C.; HULST, J. M. National malnutrition screening days in hospitalised children in The Netherlands. Archives of Disease in Childhood, [S.I.], v. 95, n. 2, p. 141-145, 2010.

KARATEKE, F.; IKIZ, G. Z.; KUVVETLI, A.; MENEKSE, E.; DAS, K.; OZYAZICI, S.; ATALAY, B. G.; OZDOGAN, M. Evaluation of nutritional risk screening-2002 and subjective global assessment for general surgery patients: a prospective study. Journal of the Pakistan Medical Association, [S.I.], v. 63, n. 11, p. 1405- 1408, 2013. 
LEANDRO-MERHI, V. A.; MÔNACO, D. V.; LAZARINI, A. L. G.; YAMASHIRO, A.; MACIEL, A. C. Estado nutricional de pacientes hospitalizados em um hospital privado. Revista Brasileira de Nutrição Clínica, Porto Alegre, v. 19, n. 3, p. 116-122, 2004.

LIMA, A. L. L.; SILVA, A. C. F.; KONNO, S. C.; CONDE, W. L.; BENICIO, M. H. D; MONTEIRO, C. A. Causas do declínio acelerado da desnutrição infantil no Nordeste do Brasil (1986-19962006). Revista de Saúde Pública, [S.I.], v. 44, n. 1, p. 17-27, 2010.

LIMA, C. M.; TIENGO, A. Perfil nutricional e alimentar de crianças internadas no Hospital das Clínicas Samuel Libânio. Revista de Ciências da Saúde, [S.I], v. 2, n. 2, p. 45-56, 2012.

MAGALHÃES, E. A.; MARTINS, M. A. L. F.; RODRIGUES, C. C.; MOREIRA, A. S. B. Associação entre tempo de internação e evolução do estado nutricional de crianças internadas em um hospital universitário. Demetra: Alimentação, Nutrição e Saúde, Rio de Janeiro, v. 8, n. 2, p. 103-114, 2013. 